\title{
Solvent Effect on Conical Intersections in Excited-State 9H-Adenine: Radiationless Decay Mechanism in Polar Solvent Supporting Information
}

\author{
Shohei Yamazaki and Shigeki Kato* \\ Department of Chemistry, Graduate School of Science, \\ Kyoto University, Kitashirakawa, Sakyo-ku, Kyoto 606-8502, Japan \\ E-mail: shigeki@kuchem.kyoto-u.ac.jp
}

Complete reference 46

(46) Werner, H.-J.; Knowles, P. J.; Lindh, R.; Schütz, M.; Celani, P.; Korona, T.; Manby, F. R.; Rauhut, G.; Amos, R. D.; Bernhardsson, A.; Berning, A.; Cooper, D. L.; Deegan, M. J. O.; Dobbyn, A. J.; Eckert, F.; Hampel, C.; Hetzer, G.; Lloyd, A. W.; McNicholas, S. J.; Meyer, W.; Mura, M. E.; Nicklass, A.; Palmieri, P.; Pitzer, R.; Schumann, U.; Stoll, H.; Stone, A. J.; Tarroni, R.; Thorsteinsson, T. MOLPRO, version 2002.6; Birmingham, UK, 2003; see http://www.molpro.net. 
Table SI1: Active spaces used for optimizations of energy minima and conical intersections

\begin{tabular}{cc}
\hline structure & active space \\
\hline$(\mathrm{gs})_{\min }\left(C_{1}\right.$ and $\left.C_{s}\right)$ & SS-CAS $(12,10)(10 \pi)^{a}$ \\
$\left({ }^{1} \mathrm{~L}_{b}\right)_{\min }\left(C_{1}\right.$ and $\left.C_{s}\right)$ & SA2-CAS $(12,10)(10 \pi)$ \\
$\left({ }^{1} \mathrm{n} \pi^{*}\right)_{\min }\left(C_{1}\right)$ & SA2-CAS $(12,10)(8 \pi 2 \mathrm{n})$ \\
$\left({ }^{1} \mathrm{~L}_{a}\right)_{\min }\left(C_{s}\right)$ & SA3-CAS $(12,10)(10 \pi)$ \\
$\left(\mathrm{gs} /{ }^{1} \mathrm{~L}_{a}\right)_{\mathrm{CI}}\left(C_{1}\right)$ & SA2-CAS $(12,10)(8 \pi 2 \mathrm{n})$ \\
$\left(\mathrm{gs} /{ }^{1} \mathrm{n} \pi^{*}\right)_{\mathrm{CI}}\left(C_{1}\right)$ & SA2-CAS $(12,10)(8 \pi 2 \mathrm{n})$ \\
$\left({ }^{1} \mathrm{~L}_{a} /{ }^{1} \mathrm{~L}_{b}\right)_{\mathrm{CI}}\left(C_{1}\right)$ & SA3-CAS $(10,9)(9 \pi)$ \\
$\left({ }^{1} \mathrm{~L}_{a} /{ }^{1} \mathrm{~L}_{b}\right)_{\mathrm{CI}}\left(C_{s}\right)$ & SA3-CAS $(12,10)(10 \pi)$ \\
$\left({ }^{1} \mathrm{~L}_{a} /{ }^{1} \mathrm{n} \pi^{*}\right)_{\mathrm{CI}}\left(C_{1}\right)$ & SA3-CAS $(12,10)(8 \pi 2 \mathrm{n})$ \\
$\left({ }^{1} \mathrm{~L}_{a} /{ }^{1} \pi \sigma^{*}\right)_{\mathrm{CI}}\left(C_{s}\right)$ & SA4-CAS $(12,11)(10 \pi 1 \sigma)$ \\
$\left({ }^{1} \mathrm{~L}_{b} /{ }^{1} \mathrm{n} \pi^{*}\right)_{\mathrm{CI}}\left(C_{1}\right.$ and $\left.C_{s}\right)$ & SA3-CAS $(12,10)(8 \pi 2 \mathrm{n})$ \\
\hline
\end{tabular}

a SS denotes "state-specific." 
Table SI2: Cartesian coordinates of energy minima (in au)

\begin{tabular}{|c|c|c|c|c|c|c|c|}
\hline & \multicolumn{3}{|c|}{$(\mathrm{gs})_{\min }\left(C_{1}\right)$} & & \multicolumn{3}{|c|}{$(\mathrm{gs})_{\min }\left(C_{s}\right)$} \\
\hline & $\mathrm{x}$ & $\mathrm{y}$ & $\mathrm{z}$ & & $\mathrm{x}$ & $\mathrm{y}$ & $\mathrm{z}$ \\
\hline & \multicolumn{3}{|c|}{ gas } & & \multicolumn{3}{|c|}{ gas } \\
\hline $\mathrm{N}_{1}$ & -3.680672658 & 0.868802722 & -0.018887556 & $\mathrm{~N}_{1}$ & -3.686237631 & 0.859790762 & 0.000000000 \\
\hline $\mathrm{C}_{2}$ & -2.508727260 & 3.122921931 & -0.008630425 & $\mathrm{C}_{2}$ & -2.516750346 & 3.115255641 & 0.000000000 \\
\hline $\mathrm{N}_{3}$ & -0.048922990 & 3.582516860 & 0.008073377 & $\mathrm{~N}_{3}$ & -0.059294937 & 3.583568950 & 0.000000000 \\
\hline $\mathrm{C}_{4}$ & 1.312051451 & 1.460295637 & 0.008449300 & $\mathrm{C}_{4}$ & 1.306885835 & 1.462320046 & 0.000000000 \\
\hline $\mathrm{C}_{5}$ & 0.355384524 & -0.980247322 & 0.001696078 & $\mathrm{C}_{5}$ & 0.358473290 & -0.978634187 & 0.000000000 \\
\hline $\mathrm{C}_{6}$ & -2.295659190 & -1.218874318 & -0.005718124 & $\mathrm{C}_{6}$ & -2.294789093 & -1.225776423 & 0.000000000 \\
\hline $\mathrm{N}_{7}$ & 2.293930897 & -2.737974574 & -0.012107372 & $\mathrm{~N}_{7}$ & 2.299487354 & -2.732800715 & 0.000000000 \\
\hline $\mathrm{C}_{8}$ & 4.339554845 & -1.375992764 & -0.008801592 & $\mathrm{C}_{8}$ & 4.342814078 & -1.366270518 & 0.000000000 \\
\hline $\mathrm{N}_{9}$ & 3.883165574 & 1.180456830 & 0.007017452 & $\mathrm{~N}_{9}$ & 3.879092687 & 1.188306577 & 0.000000000 \\
\hline $\mathrm{N}_{10}$ & -3.471866685 & -3.516715971 & 0.087448978 & $\mathrm{~N}_{10}$ & -3.450321119 & -3.512091843 & 0.000000000 \\
\hline $\mathrm{H}_{11}$ & -3.732281802 & 4.744571266 & -0.017940949 & $\mathrm{H}_{11}$ & -3.744856519 & 4.733653508 & 0.000000000 \\
\hline $\mathrm{H}_{12}$ & 6.222826653 & -2.119311868 & -0.016462711 & $\mathrm{H}_{12}$ & 6.227775552 & -2.104964688 & 0.000000000 \\
\hline $\mathrm{H}_{13}$ & 5.156990384 & 2.560508738 & 0.012840198 & $\mathrm{H}_{13}$ & 5.149185728 & 2.571635681 & 0.000000000 \\
\hline $\mathrm{H}_{14}$ & -2.454782331 & -5.014704695 & -0.419169205 & $\mathrm{H}_{14}$ & -2.431426946 & -5.085546258 & 0.000000000 \\
\hline \multirow[t]{2}{*}{$\mathrm{H}_{15}$} & -5.288488509 & -3.527766938 & -0.398511186 & $\mathrm{H}_{15}$ & -5.323821432 & -3.591705629 & 0.000000000 \\
\hline & \multicolumn{3}{|c|}{$\mathrm{CH}_{3} \mathrm{CN}$} & & \multicolumn{3}{|c|}{$\mathrm{CH}_{3} \mathrm{CN}$} \\
\hline $\mathrm{N}_{1}$ & -3.678678951 & 0.882213691 & -0.006030541 & $\mathrm{~N}_{1}$ & -3.688888310 & 0.865463354 & 0.000000000 \\
\hline $\mathrm{C}_{2}$ & -2.496323856 & 3.132802630 & -0.003308621 & $\mathrm{C}_{2}$ & -2.511545134 & 3.118085935 & 0.000000000 \\
\hline $\mathrm{N}_{3}$ & -0.036032447 & 3.584449976 & -0.002851180 & $\mathrm{~N}_{3}$ & -0.055693355 & 3.586458167 & 0.000000000 \\
\hline $\mathrm{C}_{4}$ & 1.325587006 & 1.458027348 & 0.005045990 & $\mathrm{C}_{4}$ & 1.315801672 & 1.460971943 & 0.000000000 \\
\hline $\mathrm{C}_{5}$ & 0.350948955 & -0.980623991 & 0.015451438 & $\mathrm{C}_{5}$ & 0.356635390 & -0.978440950 & 0.000000000 \\
\hline $\mathrm{C}_{6}$ & -2.299222361 & -1.211243180 & -0.003904460 & $\mathrm{C}_{6}$ & -2.298835206 & -1.226563086 & 0.000000000 \\
\hline $\mathrm{N}_{7}$ & 2.280559771 & -2.745695034 & 0.002548684 & $\mathrm{~N}_{7}$ & 2.292493787 & -2.735772233 & 0.000000000 \\
\hline $\mathrm{C}_{8}$ & 4.333745621 & -1.385848712 & -0.009013839 & $\mathrm{C}_{8}$ & 4.340785688 & -1.367660228 & 0.000000000 \\
\hline $\mathrm{N}_{9}$ & 3.888315603 & 1.165033205 & 0.000627620 & $\mathrm{~N}_{9}$ & 3.881068898 & 1.180602782 & 0.000000000 \\
\hline $\mathrm{N}_{10}$ & -3.492862668 & -3.518585689 & 0.092734932 & $\mathrm{~N}_{10}$ & -3.453049687 & -3.507499610 & 0.000000000 \\
\hline $\mathrm{H}_{11}$ & -3.715444257 & 4.758799693 & -0.011318659 & $\mathrm{H}_{11}$ & -3.739002310 & 4.738588680 & 0.000000000 \\
\hline $\mathrm{H}_{12}$ & 6.217314216 & -2.125903608 & -0.019526194 & $\mathrm{H}_{12}$ & 6.227740481 & -2.099054917 & 0.000000000 \\
\hline $\mathrm{H}_{13}$ & 5.191254854 & 2.525158135 & -0.050495462 & $\mathrm{H}_{13}$ & 5.176598149 & 2.548405563 & 0.000000000 \\
\hline $\mathrm{H}_{14}$ & -2.478772414 & -4.951869746 & -0.598502536 & $\mathrm{H}_{14}$ & -2.440636617 & -5.086977053 & 0.000000000 \\
\hline $\mathrm{H}_{15}$ & -5.255471414 & -3.488226228 & -0.580439901 & $\mathrm{H}_{15}$ & -5.327401845 & -3.606365517 & 0.000000000 \\
\hline \multicolumn{4}{|c|}{$\mathrm{H}_{2} \mathrm{O}$} & & \multicolumn{3}{|c|}{$\mathrm{H}_{2} \mathrm{O}$} \\
\hline $\mathrm{N}_{1}$ & -3.676389802 & 0.896544130 & 0.039605102 & $\mathrm{~N}_{1}$ & -3.687131674 & 0.880966124 & 0.000000000 \\
\hline $\mathrm{C}_{2}$ & -2.486093894 & 3.146331842 & 0.008460441 & $\mathrm{C}_{2}$ & -2.499404690 & 3.130976412 & 0.000000000 \\
\hline $\mathrm{N}_{3}$ & -0.018745704 & 3.574946070 & -0.009136675 & $\mathrm{~N}_{3}$ & -0.038460766 & 3.583624494 & 0.000000000 \\
\hline $\mathrm{C}_{4}$ & 1.333336914 & 1.443036953 & -0.033424617 & $\mathrm{C}_{4}$ & 1.322097782 & 1.443249669 & 0.000000000 \\
\hline $\mathrm{C}_{5}$ & 0.342148922 & -0.985893502 & 0.004406956 & $\mathrm{C}_{5}$ & 0.351133976 & -0.989026650 & 0.000000000 \\
\hline $\mathrm{C}_{6}$ & -2.301697676 & -1.191595508 & -0.005434717 & $\mathrm{C}_{6}$ & -2.310003101 & -1.231779816 & 0.000000000 \\
\hline $\mathrm{N}_{7}$ & 2.278251076 & -2.752057740 & 0.072294962 & $\mathrm{~N}_{7}$ & 2.297427369 & -2.742455873 & 0.000000000 \\
\hline $\mathrm{C}_{8}$ & 4.343731213 & -1.385186300 & -0.007512617 & $\mathrm{C}_{8}$ & 4.352940677 & -1.364673117 & 0.000000000 \\
\hline $\mathrm{N}_{9}$ & 3.896327214 & 1.147701285 & 0.000330390 & $\mathrm{~N}_{9}$ & 3.883452148 & 1.165849740 & 0.000000000 \\
\hline $\mathrm{N}_{10}$ & -3.524106027 & -3.521356675 & 0.063916735 & $\mathrm{~N}_{10}$ & -3.486845520 & -3.484110303 & 0.000000000 \\
\hline $\mathrm{H}_{11}$ & -3.696384856 & 4.776458857 & 0.003667161 & $\mathrm{H}_{11}$ & -3.717800082 & 4.757068121 & 0.000000000 \\
\hline $\mathrm{H}_{12}$ & 6.224757145 & -2.132935411 & -0.031568159 & $\mathrm{H}_{12}$ & 6.239746544 & -2.096658223 & 0.000000000 \\
\hline $\mathrm{H}_{13}$ & 5.203062860 & 2.474230250 & -0.328306562 & $\mathrm{H}_{13}$ & 5.199009972 & 2.520182463 & 0.000000000 \\
\hline $\mathrm{H}_{14}$ & -2.598035176 & -4.848397558 & -0.934242328 & $\mathrm{H}_{14}$ & -2.521785579 & -5.094824577 & 0.000000000 \\
\hline $\mathrm{H}_{15}$ & -5.290519217 & -3.412510621 & -0.631135699 & $\mathrm{H}_{15}$ & -5.363695024 & -3.584069958 & 0.000000000 \\
\hline
\end{tabular}


Table SI2: Cartesian coordinates of energy minima (in au; continued)

\begin{tabular}{|c|c|c|c|c|c|c|c|}
\hline & \multicolumn{3}{|c|}{$\left({ }^{1} \mathrm{~L}_{b}\right)_{\min }\left(C_{1}\right)$} & & \multicolumn{3}{|c|}{$\left({ }^{1} \mathrm{~L}_{b}\right)_{\min }\left(C_{s}\right)$} \\
\hline & $\mathrm{x}$ & $\mathrm{y}$ & $\mathrm{Z}$ & & $\mathrm{x}$ & $\mathrm{y}$ & $\mathrm{z}$ \\
\hline & \multicolumn{3}{|c|}{ gas } & & \multicolumn{3}{|c|}{ gas } \\
\hline $\mathrm{N}_{1}$ & -3.753194612 & 0.926230690 & -0.017708106 & $\mathrm{~N}_{1}$ & -3.769731661 & 0.897149671 & 0.000000000 \\
\hline $\mathrm{C}_{2}$ & -2.510319847 & 3.224940189 & -0.009541572 & $\mathrm{C}_{2}$ & -2.540790600 & 3.205482795 & 0.000000000 \\
\hline $\mathrm{N}_{3}$ & 0.056035490 & 3.633547788 & 0.009117270 & $\mathrm{~N}_{3}$ & 0.028804734 & 3.623475999 & 0.000000000 \\
\hline $\mathrm{C}_{4}$ & 1.382616738 & 1.496903961 & 0.016255627 & $\mathrm{C}_{4}$ & 1.371805685 & 1.505655062 & 0.000000000 \\
\hline $\mathrm{C}_{5}$ & 0.354345866 & -1.027655736 & -0.002767929 & $\mathrm{C}_{5}$ & 0.362507093 & -1.026050516 & 0.000000000 \\
\hline $\mathrm{C}_{6}$ & -2.312723301 & -1.251285847 & -0.039802747 & $\mathrm{C}_{6}$ & -2.299741698 & -1.259238741 & 0.000000000 \\
\hline $\mathrm{N}_{7}$ & 2.248234071 & -2.776149306 & -0.022103516 & $\mathrm{~N}_{7}$ & 2.265484587 & -2.763731262 & 0.000000000 \\
\hline $\mathrm{C}_{8}$ & 4.358179839 & -1.453489402 & -0.008897046 & $\mathrm{C}_{8}$ & 4.367890966 & -1.427104663 & 0.000000000 \\
\hline $\mathrm{N}_{9}$ & 3.951384646 & 1.126192821 & 0.013859788 & $\mathrm{~N}_{9}$ & 3.947984673 & 1.149677381 & 0.000000000 \\
\hline $\mathrm{N}_{10}$ & -3.580297104 & -3.512474307 & 0.117708627 & $\mathrm{~N}_{10}$ & -3.537982353 & -3.509750861 & 0.000000000 \\
\hline $\mathrm{H}_{11}$ & -3.690877083 & 4.868768424 & -0.006539472 & $\mathrm{H}_{11}$ & -3.726749642 & 4.844870838 & 0.000000000 \\
\hline $\mathrm{H}_{12}$ & 6.217481577 & -2.247199488 & -0.013161108 & $\mathrm{H}_{12}$ & 6.231454111 & -2.211071360 & 0.000000000 \\
\hline $\mathrm{H}_{13}$ & 5.268998413 & 2.463288238 & 0.027837574 & $\mathrm{H}_{13}$ & 5.258661068 & 2.493140740 & 0.000000000 \\
\hline $\mathrm{H}_{14}$ & -2.608788082 & -5.038274947 & -0.404359047 & $\mathrm{H}_{14}$ & -2.578900905 & -5.121193337 & 0.000000000 \\
\hline \multirow[t]{2}{*}{$\mathrm{H}_{15}$} & -5.367617242 & -3.462134128 & -0.472258641 & $\mathrm{H}_{15}$ & -5.413284803 & -3.525167797 & 0.000000000 \\
\hline & \multicolumn{3}{|c|}{$\mathrm{CH}_{3} \mathrm{CN}$} & & \multicolumn{3}{|c|}{$\mathrm{CH}_{3} \mathrm{CN}$} \\
\hline $\mathrm{N}_{1}$ & -3.745476467 & 0.950007711 & 0.009663846 & $\mathrm{~N}_{1}$ & -3.775639465 & 0.900298524 & 0.000000000 \\
\hline $\mathrm{C}_{2}$ & -2.486178803 & 3.238887087 & -0.002344214 & $\mathrm{C}_{2}$ & -2.538425456 & 3.204870663 & 0.000000000 \\
\hline $\mathrm{N}_{3}$ & 0.080086635 & 3.639574147 & -0.017293432 & $\mathrm{~N}_{3}$ & 0.034362822 & 3.620295140 & 0.000000000 \\
\hline $\mathrm{C}_{4}$ & 1.398251623 & 1.491550243 & 0.018839395 & $\mathrm{C}_{4}$ & 1.379491637 & 1.506392587 & 0.000000000 \\
\hline $\mathrm{C}_{5}$ & 0.346119246 & -1.024438933 & 0.033521287 & $\mathrm{C}_{5}$ & 0.359758598 & -1.023548956 & 0.000000000 \\
\hline $\mathrm{C}_{6}$ & -2.324147003 & -1.240937424 & -0.024410925 & $\mathrm{C}_{6}$ & -2.303266057 & -1.257844392 & 0.000000000 \\
\hline $\mathrm{N}_{7}$ & 2.230380179 & -2.788039930 & 0.005342580 & $\mathrm{~N}_{7}$ & 2.261617456 & -2.766621328 & 0.000000000 \\
\hline $\mathrm{C}_{8}$ & 4.347847778 & -1.473179543 & -0.016480921 & $\mathrm{C}_{8}$ & 4.365950553 & -1.427120797 & 0.000000000 \\
\hline $\mathrm{N}_{9}$ & 3.956739647 & 1.101889090 & 0.001736851 & $\mathrm{~N}_{9}$ & 3.951944725 & 1.142851891 & 0.000000000 \\
\hline $\mathrm{N}_{10}$ & -3.612972659 & -3.511850454 & 0.093683215 & $\mathrm{~N}_{10}$ & -3.540470889 & -3.501782065 & 0.000000000 \\
\hline $\mathrm{H}_{11}$ & -3.660743327 & 4.888241646 & -0.020548893 & $\mathrm{H}_{11}$ & -3.720668430 & 4.848055268 & 0.000000000 \\
\hline $\mathrm{H}_{12}$ & 6.206370726 & -2.267438488 & -0.033286699 & $\mathrm{H}_{12}$ & 6.231736124 & -2.204997137 & 0.000000000 \\
\hline $\mathrm{H}_{13}$ & 5.302757649 & 2.416585606 & -0.082279264 & $\mathrm{H}_{13}$ & 5.287109731 & 2.469801993 & 0.000000000 \\
\hline $\mathrm{H}_{14}$ & -2.628530845 & -4.977122917 & -0.579071786 & $\mathrm{H}_{14}$ & -2.592855650 & -5.122381063 & 0.000000000 \\
\hline $\mathrm{H}_{15}$ & -5.331077367 & -3.425088513 & -0.6877566655 & $\mathrm{H}_{15}$ & -5.417892118 & -3.532908727 & 0.000000000 \\
\hline \multicolumn{4}{|c|}{$\mathrm{H}_{2} \mathrm{O}$} & & \multicolumn{3}{|c|}{$\mathrm{H}_{2} \mathrm{O}$} \\
\hline $\mathrm{N}_{1}$ & -3.734397438 & 0.971956427 & 0.083030044 & $\mathrm{~N}_{1}$ & -3.786329665 & 0.895817245 & 0.000000000 \\
\hline $\mathrm{C}_{2}$ & -2.465702764 & 3.252892275 & -0.009898516 & $\mathrm{C}_{2}$ & -2.545829473 & 3.205193412 & 0.000000000 \\
\hline $\mathrm{N}_{3}$ & 0.103493208 & 3.643182814 & -0.026374369 & $\mathrm{~N}_{3}$ & 0.040916598 & 3.603734573 & 0.000000000 \\
\hline $\mathrm{C}_{4}$ & 1.407034007 & 1.472154890 & -0.020826665 & $\mathrm{C}_{4}$ & 1.380801350 & 1.498023248 & 0.000000000 \\
\hline $\mathrm{C}_{5}$ & 0.336377819 & -1.020282977 & 0.048443741 & $\mathrm{C}_{5}$ & 0.355796026 & -1.028253801 & 0.000000000 \\
\hline $\mathrm{C}_{6}$ & -2.332854394 & -1.218927468 & 0.055444609 & $\mathrm{C}_{6}$ & -2.306586815 & -1.261339123 & 0.000000000 \\
\hline $\mathrm{N}_{7}$ & 2.223297929 & -2.794455085 & 0.108955187 & $\mathrm{~N}_{7}$ & 2.275632222 & -2.765962722 & 0.000000000 \\
\hline $\mathrm{C}_{8}$ & 4.347668194 & -1.484651369 & -0.019768010 & $\mathrm{C}_{8}$ & 4.378359379 & -1.411400706 & 0.000000000 \\
\hline $\mathrm{N}_{9}$ & 3.962700804 & 1.079612022 & -0.019362949 & $\mathrm{~N}_{9}$ & 3.956238066 & 1.140478589 & 0.000000000 \\
\hline $\mathrm{N}_{10}$ & -3.643272928 & -3.528667387 & -0.005417232 & $\mathrm{~N}_{10}$ & -3.551867039 & -3.479910512 & 0.000000000 \\
\hline $\mathrm{H}_{11}$ & -3.640897699 & 4.897078736 & -0.114940721 & $\mathrm{H}_{11}$ & -3.717220555 & 4.855104372 & 0.000000000 \\
\hline $\mathrm{H}_{12}$ & 6.201428343 & -2.289008835 & -0.100522291 & $\mathrm{H}_{12}$ & 6.246236598 & -2.186200548 & 0.000000000 \\
\hline $\mathrm{H}_{13}$ & 5.283007819 & 2.338996264 & -0.518068870 & $\mathrm{H}_{13}$ & 5.309264375 & 2.454032404 & 0.000000000 \\
\hline $\mathrm{H}_{14}$ & -2.746718804 & -4.820836048 & -1.085924889 & $\mathrm{H}_{14}$ & -2.644847643 & -5.127513756 & 0.000000000 \\
\hline $\mathrm{H}_{15}$ & -5.377296045 & -3.324664780 & -0.773854696 & $\mathrm{H}_{15}$ & -5.433028230 & -3.519285089 & 0.000000000 \\
\hline
\end{tabular}


Table SI2: Cartesian coordinates of energy minima (in au; continued)

\begin{tabular}{|c|c|c|c|c|c|c|c|}
\hline & \multicolumn{3}{|c|}{$\left({ }^{1} \mathrm{n} \pi^{*}\right)_{\min }\left(C_{1}\right)$} & & \multicolumn{3}{|c|}{$\left({ }^{1} \mathrm{~L}_{a}\right)_{\min }\left(C_{s}\right)$} \\
\hline & $\mathrm{x}$ & $\mathrm{y}$ & $\mathrm{z}$ & & $\mathrm{x}$ & $\mathrm{y}$ & $\mathrm{z}$ \\
\hline & \multicolumn{3}{|c|}{ gas } & & \multicolumn{3}{|c|}{ gas } \\
\hline $\mathrm{N}_{1}$ & -3.631863523 & 0.725983274 & -0.192842318 & $\mathrm{~N}_{1}$ & -3.738005309 & 0.934413590 & 0.000000000 \\
\hline $\mathrm{C}_{2}$ & -2.822724265 & 3.148487807 & 0.478031545 & $\mathrm{C}_{2}$ & -2.424009490 & 3.228577099 & 0.000000000 \\
\hline $\mathrm{N}_{3}$ & -0.261777911 & 3.484675589 & -0.221931985 & $\mathrm{~N}_{3}$ & 0.235801025 & 3.688474057 & 0.000000000 \\
\hline $\mathrm{C}_{4}$ & 1.255372612 & 1.504700037 & -0.060601727 & $\mathrm{C}_{4}$ & 1.423883693 & 1.515722888 & 0.000000000 \\
\hline $\mathrm{C}_{5}$ & 0.436677239 & -1.002012663 & 0.052254038 & $\mathrm{C}_{5}$ & 0.320761801 & -1.010592939 & 0.000000000 \\
\hline $\mathrm{C}_{6}$ & -2.214899285 & -1.330705881 & -0.038600653 & $\mathrm{C}_{6}$ & -2.462377776 & -1.139412042 & 0.000000000 \\
\hline $\mathrm{N}_{7}$ & 2.471352582 & -2.648357317 & 0.119490208 & $\mathrm{~N}_{7}$ & 2.115436575 & -2.813146402 & 0.000000000 \\
\hline $\mathrm{C}_{8}$ & 4.440580567 & -1.197250044 & 0.053938778 & $\mathrm{C}_{8}$ & 4.287402962 & -1.586703652 & 0.000000000 \\
\hline $\mathrm{N}_{9}$ & 3.834008294 & 1.365258749 & -0.083293645 & $\mathrm{~N}_{9}$ & 3.941278782 & 1.045125778 & 0.000000000 \\
\hline $\mathrm{N}_{10}$ & -3.345134055 & -3.661736503 & 0.035836658 & $\mathrm{~N}_{10}$ & -3.533450547 & -3.446507612 & 0.000000000 \\
\hline $\mathrm{H}_{11}$ & -4.076341193 & 4.666802082 & -0.001828666 & $\mathrm{H}_{11}$ & -3.591555684 & 4.879561111 & 0.000000000 \\
\hline $\mathrm{H}_{12}$ & 6.362866541 & -1.827929082 & 0.074688170 & $\mathrm{H}_{12}$ & 6.118888717 & -2.438190735 & 0.000000000 \\
\hline $\mathrm{H}_{13}$ & 5.033798412 & 2.806504360 & -0.029796727 & $\mathrm{H}_{13}$ & 5.287816212 & 2.356029209 & 0.000000000 \\
\hline $\mathrm{H}_{14}$ & -2.265681358 & -5.102053179 & -0.513273665 & $\mathrm{H}_{14}$ & -2.456385802 & -4.984706365 & 0.000000000 \\
\hline \multirow[t]{2}{*}{$\mathrm{H}_{15}$} & -5.132096451 & -3.725657050 & -0.546642772 & $\mathrm{H}_{15}$ & -5.407203853 & -3.597886307 & 0.000000000 \\
\hline & \multicolumn{3}{|c|}{$\mathrm{CH}_{3} \mathrm{CN}$} & & \multicolumn{3}{|c|}{$\mathrm{CH}_{3} \mathrm{CN}$} \\
\hline $\mathrm{N}_{1}$ & -3.712153495 & 0.686485720 & -0.072274263 & $\mathrm{~N}_{1}$ & -3.738487549 & 0.929679803 & 0.000000000 \\
\hline $\mathrm{C}_{2}$ & -2.871493710 & 3.122889641 & 0.444076853 & $\mathrm{C}_{2}$ & -2.427245168 & 3.223755329 & 0.000000000 \\
\hline $\mathrm{N}_{3}$ & -0.313375679 & 3.430876778 & -0.279406829 & $\mathrm{~N}_{3}$ & 0.219760668 & 3.701137974 & 0.000000000 \\
\hline $\mathrm{C}_{4}$ & 1.300804914 & 1.506663269 & -0.075167078 & $\mathrm{C}_{4}$ & 1.425001886 & 1.513707738 & 0.000000000 \\
\hline $\mathrm{C}_{5}$ & 0.453727933 & -0.965881436 & 0.057629419 & $\mathrm{C}_{5}$ & 0.318987441 & -1.005272281 & 0.000000000 \\
\hline $\mathrm{C}_{6}$ & -2.237623144 & -1.300079104 & -0.035176499 & $\mathrm{C}_{6}$ & -2.458760904 & -1.149645833 & 0.000000000 \\
\hline $\mathrm{N}_{7}$ & 2.486280498 & -2.613447347 & 0.137273385 & $\mathrm{~N}_{7}$ & 2.126582638 & -2.812343220 & 0.000000000 \\
\hline $\mathrm{C}_{8}$ & 4.466859573 & -1.167780543 & 0.066454564 & $\mathrm{C}_{8}$ & 4.281978551 & -1.575561416 & 0.000000000 \\
\hline $\mathrm{N}_{9}$ & 3.865777435 & 1.385514134 & -0.068323619 & $\mathrm{~N}_{9}$ & 3.927107662 & 1.051896924 & 0.000000000 \\
\hline $\mathrm{N}_{10}$ & -3.286692404 & -3.694321643 & -0.002182170 & $\mathrm{~N}_{10}$ & -3.512535404 & -3.458239206 & 0.000000000 \\
\hline $\mathrm{H}_{11}$ & -4.118322780 & 4.648137968 & -0.028283852 & $\mathrm{H}_{11}$ & -3.605804088 & 4.870328926 & 0.000000000 \\
\hline $\mathrm{H}_{12}$ & 6.389697405 & -1.792770994 & 0.098070800 & $\mathrm{H}_{12}$ & 6.125084479 & -2.402026648 & 0.000000000 \\
\hline $\mathrm{H}_{13}$ & 5.082322716 & 2.822112990 & -0.081707052 & $\mathrm{H}_{13}$ & 5.297876963 & 2.347855451 & 0.000000000 \\
\hline $\mathrm{H}_{14}$ & -2.206308056 & -5.000147916 & -0.834525218 & $\mathrm{H}_{14}$ & -2.431241264 & -4.996385598 & 0.000000000 \\
\hline $\mathrm{H}_{15}$ & -5.058909510 & -3.742008572 & -0.649819571 & $\mathrm{H}_{15}$ & -5.385443560 & -3.650151315 & 0.000000000 \\
\hline \multicolumn{4}{|c|}{$\mathrm{H}_{2} \mathrm{O}$} & & \multicolumn{3}{|c|}{$\mathrm{H}_{2} \mathrm{O}$} \\
\hline $\mathrm{N}_{1}$ & -3.462410335 & 0.850496155 & -0.017889860 & $\mathrm{~N}_{1}$ & -3.720843217 & 0.928362769 & 0.000000000 \\
\hline $\mathrm{C}_{2}$ & -2.686423114 & 3.335982519 & 0.143982661 & $\mathrm{C}_{2}$ & -2.437314744 & 3.234255448 & 0.000000000 \\
\hline $\mathrm{N}_{3}$ & -0.027279389 & 3.590571552 & -0.019064137 & $\mathrm{~N}_{3}$ & 0.198692068 & 3.723554111 & 0.000000000 \\
\hline $\mathrm{C}_{4}$ & 1.279591947 & 1.471380909 & -0.003339891 & $\mathrm{C}_{4}$ & 1.426325478 & 1.490695621 & 0.000000000 \\
\hline $\mathrm{C}_{5}$ & 0.357092428 & -1.019390915 & 0.176147071 & $\mathrm{C}_{5}$ & 0.321611983 & -1.008342889 & 0.000000000 \\
\hline $\mathrm{C}_{6}$ & -2.280173250 & -1.324348232 & 0.008883493 & $\mathrm{C}_{6}$ & -2.456205216 & -1.171389637 & 0.000000000 \\
\hline $\mathrm{N}_{7}$ & 2.337429083 & -2.749945532 & 0.144646706 & $\mathrm{~N}_{7}$ & 2.159837191 & -2.812788550 & 0.000000000 \\
\hline $\mathrm{C}_{8}$ & 4.357540296 & -1.358689528 & -0.059396128 & $\mathrm{C}_{8}$ & 4.292948855 & -1.562429887 & 0.000000000 \\
\hline $\mathrm{N}_{9}$ & 3.845951117 & 1.197439790 & -0.083816927 & $\mathrm{~N}_{9}$ & 3.903957050 & 1.066258414 & 0.000000000 \\
\hline $\mathrm{N}_{10}$ & -3.536953866 & -3.611746936 & -0.019594294 & $\mathrm{~N}_{10}$ & -3.521919274 & -3.471629011 & 0.000000000 \\
\hline $\mathrm{H}_{11}$ & -3.846636762 & 4.721459116 & -0.791449427 & $\mathrm{H}_{11}$ & -3.635758237 & 4.869543117 & 0.000000000 \\
\hline $\mathrm{H}_{12}$ & 6.254002298 & -2.055788761 & -0.172156886 & $\mathrm{H}_{12}$ & 6.149585481 & -2.358542669 & 0.000000000 \\
\hline $\mathrm{H}_{13}$ & 5.093575898 & 2.531398694 & -0.557318360 & $\mathrm{H}_{13}$ & 5.292151981 & 2.354914803 & 0.000000000 \\
\hline $\mathrm{H}_{14}$ & -2.678274121 & -4.867922267 & -1.160266689 & $\mathrm{H}_{14}$ & -2.461771804 & -5.024399979 & 0.000000000 \\
\hline $\mathrm{H}_{15}$ & -5.349986657 & -3.446332706 & -0.551367162 & $\mathrm{H}_{15}$ & -5.394391862 & -3.684094186 & 0.000000000 \\
\hline
\end{tabular}


Table SI3: Cartesian coordinates of conical intersections (in au)

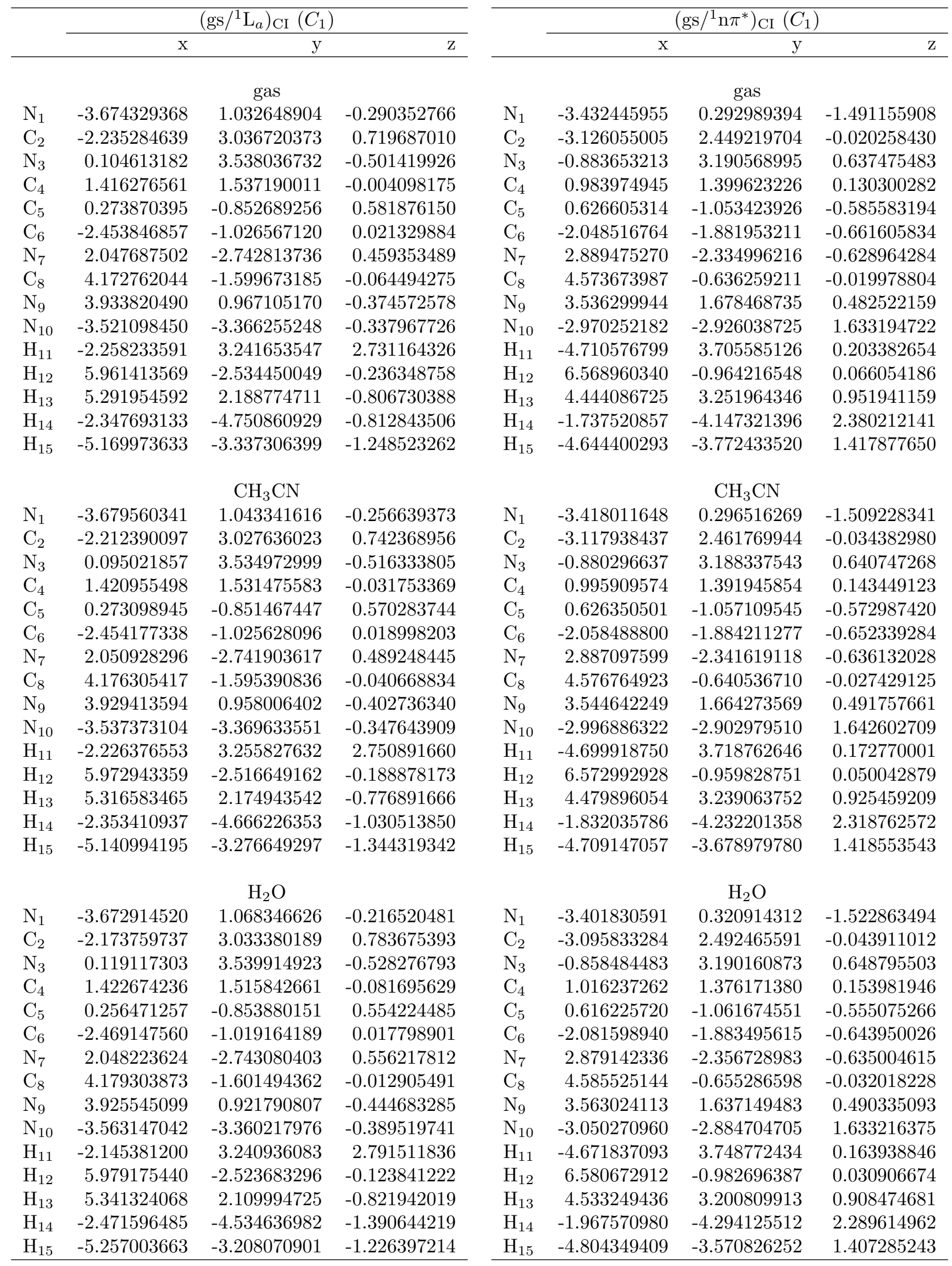


Table SI3: Cartesian coordinates of conical intersections (in au; continued)

\begin{tabular}{|c|c|c|c|}
\hline & \multicolumn{3}{|c|}{$\left({ }^{1} \mathrm{~L}_{a} /{ }^{1} \mathrm{~L}_{b}\right)_{\mathrm{CI}}\left(C_{1}\right)$} \\
\hline & $\mathrm{x}$ & $\mathrm{y}$ & $\mathrm{Z}$ \\
\hline & \multicolumn{3}{|c|}{ gas } \\
\hline $\mathrm{N}_{1}$ & -3.647998262 & 0.964166414 & -0.156463120 \\
\hline $\mathrm{C}_{2}$ & -2.242240409 & 3.177680048 & 0.590406563 \\
\hline $\mathrm{N}_{3}$ & 0.120398823 & 3.689077633 & -0.388691225 \\
\hline $\mathrm{C}_{4}$ & 1.406029752 & 1.486571123 & -0.085489951 \\
\hline $\mathrm{C}_{5}$ & 0.253340583 & -0.958673503 & 0.443618547 \\
\hline $\mathrm{C}_{6}$ & -2.404887207 & -1.131009040 & 0.026915040 \\
\hline $\mathrm{N}_{7}$ & 2.150295891 & -2.794322316 & 94016 \\
\hline $\mathrm{C}_{8}$ & 220929159 & -1.616729858 & 46589 \\
\hline $\mathrm{N}_{9}$ & 884310158 & 1.044595695 & -0.291375557 \\
\hline $\mathrm{N}_{10}$ & .576154220 & -3.462438901 & -0.288670552 \\
\hline $\mathrm{H}_{11}$ & -3.356772272 & 4.718606145 & 1.269610379 \\
\hline $\mathrm{H}_{12}$ & 6.068693063 & -2.444316069 & 8797 \\
\hline $\mathrm{H}_{13}$ & 236200491 & 2.291438491 & 2296 \\
\hline $\mathrm{H}_{14}$ & 3453 & -4.858265673 & 5889 \\
\hline \multirow[t]{2}{*}{$\mathrm{H}_{15}$} & 4222 & -3.370410513 & 569 \\
\hline & \multicolumn{3}{|c|}{$\mathrm{CH}_{3} \mathrm{CN}$} \\
\hline $\mathrm{N}_{1}$ & -3.67 & 0.946728171 & 5875 \\
\hline $\mathrm{C}_{2}$ & -2.2 & 3.15342 & 166 \\
\hline $\mathrm{N}_{3}$ & 79 & 3.6789 & 021 \\
\hline $\mathrm{C}_{4}$ & 21 & 1.4942 & 1028 \\
\hline $\mathrm{C}_{5}$ & & -0.9500 & 882 \\
\hline $\mathrm{C}_{6}$ & 59 & -1.1326 & \\
\hline $\mathrm{N}_{7}$ & & -2.7 & \\
\hline $\mathrm{C}_{8}$ & & -1.590 & \\
\hline $\mathrm{N}_{9}$ & & 50 & \\
\hline $\mathrm{N}_{10}$ & & -3.4788 & 179 \\
\hline $\mathrm{H}_{1}$ & & 74 & \\
\hline $\mathrm{H}$ & & -2.4013 & 50 \\
\hline $\mathrm{H}_{13}$ & & $\$ 2$ & \\
\hline $\mathrm{H}_{14}$ & & -4 & 380 \\
\hline \multirow{2}{*}{$\mathrm{H}_{1}$} & 1556 & -3.354515374 & -1.16 \\
\hline & \multicolumn{3}{|c|}{$\mathrm{H}_{2} \mathrm{C}$} \\
\hline $\mathrm{N}_{1}$ & 7 & 88 & 6018 \\
\hline $\mathrm{C}_{2}$ & & 3.1375 & 0571 \\
\hline $\mathrm{N}_{3}$ & 755 & 3.647613863 & -0.365639502 \\
\hline $\mathrm{C}_{4}$ & & 1.496105650 & -0.140785789 \\
\hline $\mathrm{C}_{5}$ & 96873621 & -0.960942861 & 0.388365307 \\
\hline $\mathrm{C}_{6}$ & -2.414319285 & -1.147638925 & -0.005307741 \\
\hline $\mathrm{N}_{7}$ & 2.160435179 & -2.764656902 & 0.470420135 \\
\hline $\mathrm{C}_{8}$ & 155 & -1.544927344 & 09881 \\
\hline $\mathrm{N}_{9}$ & & 1.039524800 & -0.343967955 \\
\hline $\mathrm{N}_{10}$ & & -3.465395876 & ).302599915 \\
\hline $\mathrm{H}_{11}$ & & .741467372 & 46829 \\
\hline $\mathrm{H}_{12}$ & 6.122194200 & -2.352678692 & 0.009053435 \\
\hline $\mathrm{H}_{13}$ & 270080398 & 2.271998920 & -0.726989350 \\
\hline $\mathrm{H}_{14}$ & -2.484997660 & -4.772974277 & -1.158995065 \\
\hline $\mathrm{H}_{15}$ & -5.266049374 & -3.360433713 & -1.072518443 \\
\hline
\end{tabular}

\begin{tabular}{ccc}
\hline \multicolumn{3}{c}{$\left({ }^{1} \mathrm{~L}_{a} /{ }^{1} \mathrm{~L}_{b}\right)_{\mathrm{CI}}\left(C_{s}\right)$} \\
$\mathrm{x}$ & $\mathrm{y}$ & $\mathrm{z}$ \\
\hline
\end{tabular}

\begin{tabular}{lrrr} 
& \multicolumn{3}{c}{$\mathrm{x}$} \\
\hline & & gas & \\
$\mathrm{N}_{1}$ & -3.751154530 & 0.997249705 & 0.000000000 \\
$\mathrm{C}_{2}$ & -2.322215939 & 3.263063569 & 0.000000000 \\
$\mathrm{~N}_{3}$ & 0.358750287 & 3.720386279 & 0.000000000 \\
$\mathrm{C}_{4}$ & 1.478130206 & 1.496493285 & 0.000000000 \\
$\mathrm{C}_{5}$ & 0.289025354 & -0.995813462 & 0.000000000 \\
$\mathrm{C}_{6}$ & -2.537939725 & -1.080000754 & 0.000000000 \\
$\mathrm{~N}_{7}$ & 2.012795778 & -2.845963844 & 0.000000000 \\
$\mathrm{C}_{8}$ & 4.239997024 & -1.703155477 & 0.000000000 \\
$\mathrm{~N}_{9}$ & 3.959801238 & 0.968950471 & 0.000000000 \\
$\mathrm{~N}_{10}$ & -3.568786517 & -3.396673586 & 0.000000000 \\
$\mathrm{H}_{11}$ & -3.463613836 & 4.933065352 & 0.000000000 \\
$\mathrm{H}_{12}$ & 6.044672248 & -2.613063902 & 0.000000000 \\
$\mathrm{H}_{13}$ & 5.332321605 & 2.253463649 & 0.000000000 \\
$\mathrm{H}_{14}$ & -2.441615830 & -4.897140626 & 0.000000000 \\
$\mathrm{H}_{15}$ & -5.432561028 & -3.598844569 & 0.000000000
\end{tabular}

\section{$\mathrm{CH}_{3} \mathrm{CN}$}

$\begin{array}{lll}\mathrm{N}_{1} & -3.755455737 & 0.962486347\end{array}$

$\mathrm{C}_{2} \quad-2.372538553 \quad 3.237836684$

$\mathrm{N}_{3} \quad 0.281599504 \quad 3.728234684$

$\mathrm{C}_{4} \quad 1.451349413$

$\mathrm{C}_{5} \quad 0.296065291$

$\mathrm{C}_{6}-2.496087027$

$\mathrm{N}_{7} \quad 2.077252584$

$\mathrm{C}_{8} \quad 4.250804668$

$\mathrm{N}_{9} \quad 3.927137206$

$\mathrm{N}_{10} \quad-3.509238127$

$\mathrm{H}_{11} \quad-3.543192956$

$\mathrm{H}_{12} \quad 6.084006857$

$\mathrm{H}_{13} \quad 5.308920587$

$\mathrm{H}_{14} \quad-2.374942795$

$\mathrm{H}_{15} \quad-5.369920056$

$\mathrm{N}_{1} \quad-3.723035206$

$\mathrm{C}_{2} \quad-2.432704134$

$\mathrm{N}_{3} \quad 0.199973269$

$\mathrm{C}_{4} \quad 1.426644860$

$\mathrm{C}_{5} \quad 0.315350121$

$\mathrm{C}_{6} \quad-2.448735479$

$\mathrm{N}_{7} \quad 2.156803079$

$\mathrm{C}_{8} \quad 4.291574187$

$\mathrm{N}_{9} \quad 3.905143548$

$\mathrm{N}_{10} \quad-3.524673887$

$\mathrm{H}_{11} \quad-3.632215976$

$\mathrm{H}_{12} \quad 6.146898333$

$\mathrm{H}_{13} \quad 5.291481894$

$\mathrm{H}_{14} \quad-2.458016032$

$\mathrm{H}_{15} \quad-5.393615896$
1.506957833

$-0.991133964$

$-1.124223528$

$-2.831228724$

$-1.637749442$

1.016657803

$-3.441745830$

4.890455085

$-2.487110584$

2.300895276

$-4.941199857$

$-3.688484983$

\section{$\mathrm{H}_{2} \mathrm{O}$}

0.938510353

3.236951713

3.725268730

1.489687534

$-1.006074829$

$-1.176013853$

$-2.815410804$

$-1.566084572$

1.062730292

$-3.472423029$

4.871014965

$-2.364245247$

2.352151897

$-5.021145318$

$-3.688584192$
0.000000000 0.000000000 0.000000000 0.000000000 0.000000000 0.000000000 0.000000000 0.000000000 0.000000000 0.000000000 0.000000000 0.000000000 0.000000000 0.000000000 0.000000000

0.000000000 0.000000000 0.000000000 0.000000000 0.000000000 0.000000000 0.000000000 0.000000000 0.000000000 0.000000000 0.000000000 0.000000000 0.000000000 0.000000000 0.000000000 
Table SI3: Cartesian coordinates of conical intersections (in au; continued)

\begin{tabular}{|c|c|c|c|c|c|c|c|}
\hline & \multicolumn{3}{|c|}{$\left({ }^{1} \mathrm{~L}_{a} /{ }^{1} \mathrm{n} \pi^{*}\right)_{\mathrm{CI}}\left(C_{1}\right)$} & & \multicolumn{3}{|c|}{$\left({ }^{1} \mathrm{~L}_{a} /{ }^{1} \pi \sigma^{*}\right)_{\mathrm{CI}}\left(C_{s}\right)$} \\
\hline & $\mathrm{x}$ & $\mathrm{y}$ & $\mathrm{z}$ & & $\mathrm{x}$ & $\mathrm{y}$ & $\mathrm{z}$ \\
\hline & \multicolumn{3}{|c|}{ gas } & & \multicolumn{3}{|c|}{ gas } \\
\hline $\mathrm{N}_{1}$ & -3.607850636 & 0.883999187 & -0.372160810 & $\mathrm{~N}_{1}$ & -3.772217446 & 0.972258540 & 0.000000000 \\
\hline $\mathrm{C}_{2}$ & -2.608772920 & 3.100476221 & 0.593290451 & $\mathrm{C}_{2}$ & -2.409603088 & 3.221036065 & 0.000000000 \\
\hline $\mathrm{N}_{3}$ & -0.133036320 & 3.543424665 & -0.354539560 & $\mathrm{~N}_{3}$ & 0.215013453 & 3.712694351 & 0.000000000 \\
\hline $\mathrm{C}_{4}$ & 1.268848224 & 1.546018158 & -0.033567799 & $\mathrm{C}_{4}$ & 1.444356345 & 1.522948300 & 0.000000000 \\
\hline $\mathrm{C}_{5}$ & 0.336608251 & -0.962725081 & 0.370377163 & $\mathrm{C}_{5}$ & 0.311565938 & -0.999226854 & 0.000000000 \\
\hline $\mathrm{C}_{6}$ & -2.255947651 & -1.265113033 & 0.014631873 & $\mathrm{C}_{6}$ & -2.447972854 & -1.179306026 & 0.000000000 \\
\hline $\mathrm{N}_{7}$ & 2.332209246 & -2.701191030 & 0.328697672 & $\mathrm{~N}_{7}$ & 2.090543697 & -2.818433684 & 0.000000000 \\
\hline $\mathrm{C}_{8}$ & 4.335628642 & -1.352531250 & -0.008718546 & $\mathrm{C}_{8}$ & 4.286552252 & -1.579800594 & 0.000000000 \\
\hline $\mathrm{N}_{9}$ & 3.843065139 & 1.228877297 & -0.269226341 & $\mathrm{~N}_{9}$ & 3.933695871 & 1.019691402 & 0.000000000 \\
\hline $\mathrm{N}_{10}$ & -3.397290117 & -3.542662992 & -0.277610677 & $\mathrm{~N}_{10}$ & -3.504220068 & -3.451066846 & 0.000000000 \\
\hline $\mathrm{H}_{11}$ & -3.049887081 & 3.675836832 & 2.511746687 & $\mathrm{H}_{11}$ & -3.609222253 & 4.890138326 & 0.000000000 \\
\hline $\mathrm{H}_{12}$ & 6.228156012 & -2.071479457 & -0.085532845 & $\mathrm{H}_{12}$ & 6.115007893 & -2.475565586 & 0.000000000 \\
\hline $\mathrm{H}_{13}$ & 5.099840447 & 2.593135147 & -0.519146592 & $\mathrm{H}_{13}$ & 5.281211494 & 2.369570906 & 0.000000000 \\
\hline $\mathrm{H}_{14}$ & -2.403299410 & -5.068034961 & 0.166820915 & $\mathrm{H}_{14}$ & -2.393029063 & -4.982170081 & 0.000000000 \\
\hline \multirow[t]{2}{*}{$\mathrm{H}_{15}$} & -5.263266475 & -3.618218177 & -0.140131531 & $\mathrm{H}_{15}$ & -5.376479904 & -3.659678970 & 0.000000000 \\
\hline & \multicolumn{3}{|c|}{$\mathrm{CH}_{3} \mathrm{CN}$} & & \multicolumn{3}{|c|}{$\mathrm{CH}_{3} \mathrm{CN}$} \\
\hline $\mathrm{N}_{1}$ & -3.612408686 & 0.918843761 & -0.368364754 & $\mathrm{~N}_{1}$ & -3.734364364 & 1.015549072 & 0.000000000 \\
\hline $\mathrm{C}_{2}$ & -2.565740029 & 3.100796510 & 0.637694184 & $\mathrm{C}_{2}$ & -2.407967371 & 3.185124372 & 0.000000000 \\
\hline $\mathrm{N}_{3}$ & -0.120842715 & 3.539356143 & -0.367319294 & $\mathrm{~N}_{3}$ & 0.159620262 & 3.668380862 & 0.000000000 \\
\hline $\mathrm{C}_{4}$ & 1.294822259 & 1.527961065 & -0.053287248 & $\mathrm{C}_{4}$ & 1.427737505 & 1.509831684 & 0.000000000 \\
\hline $\mathrm{C}_{5}$ & 0.334072267 & -0.963697648 & 0.325324456 & $\mathrm{C}_{5}$ & 0.317523562 & -1.009351011 & 0.000000000 \\
\hline $\mathrm{C}_{6}$ & -2.273328278 & -1.244620481 & 0.003127984 & $\mathrm{C}_{6}$ & -2.413493305 & -1.212954153 & 0.000000000 \\
\hline $\mathrm{N}_{7}$ & 2.315367771 & -2.714291192 & 0.335780735 & $\mathrm{~N}_{7}$ & 2.096352684 & -2.807409907 & 0.000000000 \\
\hline $\mathrm{C}_{8}$ & 4.333126430 & -1.371550330 & 0.023438615 & $\mathrm{C}_{8}$ & 4.282612533 & -1.523599591 & 0.000000000 \\
\hline $\mathrm{N}_{9}$ & 3.854816031 & 1.199445606 & -0.292903197 & $\mathrm{~N}_{9}$ & 3.937151931 & 1.025136527 & 0.000000000 \\
\hline $\mathrm{N}_{10}$ & -3.444894257 & -3.518626057 & -0.305567853 & $\mathrm{~N}_{10}$ & -3.505512622 & -3.430332451 & 0.000000000 \\
\hline $\mathrm{H}_{11}$ & -2.949637201 & 3.615696366 & 2.586125377 & $\mathrm{H}_{11}$ & -3.606875769 & 4.868300626 & 0.000000000 \\
\hline $\mathrm{H}_{12}$ & 6.225579179 & -2.090862062 & -0.017557374 & $\mathrm{H}_{12}$ & 6.125994575 & -2.397207930 & 0.000000000 \\
\hline $\mathrm{H}_{13}$ & 5.149482731 & 2.560817067 & -0.346674424 & $\mathrm{H}_{13}$ & 5.296468925 & 2.351812933 & 0.000000000 \\
\hline $\mathrm{H}_{14}$ & -2.482743627 & -5.002863114 & 0.338736791 & $\mathrm{H}_{14}$ & -2.425200440 & -4.999176185 & 0.000000000 \\
\hline $\mathrm{H}_{15}$ & -5.280637646 & -3.548710352 & 0.112623834 & $\mathrm{H}_{15}$ & -5.384660545 & -3.652875636 & 0.000000000 \\
\hline \multicolumn{4}{|c|}{$\mathrm{H}_{2} \mathrm{O}$} & & \multicolumn{3}{|c|}{$\mathrm{H}_{2} \mathrm{O}$} \\
\hline $\mathrm{N}_{1}$ & -3.597608309 & 0.951979413 & -0.403654334 & $\mathrm{~N}_{1}$ & -3.717640311 & 1.014446118 & 0.000000000 \\
\hline $\mathrm{C}_{2}$ & -2.525044905 & 3.071466021 & 0.670967605 & $\mathrm{C}_{2}$ & -2.409896187 & 3.207894347 & 0.000000000 \\
\hline $\mathrm{N}_{3}$ & -0.092085227 & 3.546425115 & -0.362232849 & $\mathrm{~N}_{3}$ & 0.168945838 & 3.675690842 & 0.000000000 \\
\hline $\mathrm{C}_{4}$ & 1.303461338 & 1.515377008 & -0.055291165 & $\mathrm{C}_{4}$ & 1.428847104 & 1.505311197 & 0.000000000 \\
\hline $\mathrm{C}_{5}$ & 0.320111899 & -0.979118336 & 0.309631774 & $\mathrm{C}_{5}$ & 0.317285077 & -1.029003942 & 0.000000000 \\
\hline $\mathrm{C}_{6}$ & -2.294493874 & -1.247452930 & 0.000641838 & $\mathrm{C}_{6}$ & -2.422829488 & -1.220675814 & 0.000000000 \\
\hline $\mathrm{N}_{7}$ & 2.318689857 & -2.727838706 & 0.323365025 & $\mathrm{~N}_{7}$ & 2.125751367 & -2.817245371 & 0.000000000 \\
\hline $\mathrm{C}_{8}$ & 4.341438810 & -1.380167468 & 0.030380014 & $\mathrm{C}_{8}$ & 4.300172675 & -1.522119259 & 0.000000000 \\
\hline $\mathrm{N}_{9}$ & 3.855805152 & 1.181480495 & -0.302258929 & $\mathrm{~N}_{9}$ & 3.924602974 & 1.033722357 & 0.000000000 \\
\hline $\mathrm{N}_{10}$ & -3.497942599 & -3.468946039 & -0.264809430 & $\mathrm{~N}_{10}$ & -3.543836391 & -3.428536024 & 0.000000000 \\
\hline $\mathrm{H}_{11}$ & -2.938264530 & 3.607702544 & 2.609877984 & $\mathrm{H}_{11}$ & -3.622303410 & 4.875422796 & 0.000000000 \\
\hline $\mathrm{H}_{12}$ & 6.235099738 & -2.098451234 & 0.004607859 & $\mathrm{H}_{12}$ & 6.151871862 & -2.369641894 & 0.000000000 \\
\hline $\mathrm{H}_{13}$ & 5.171477940 & 2.530272485 & -0.281624183 & $\mathrm{H}_{13}$ & 5.296584548 & 2.344019759 & 0.000000000 \\
\hline $\mathrm{H}_{14}$ & -2.626682206 & -5.012798020 & 0.347264994 & $\mathrm{H}_{14}$ & -2.517964478 & -5.034116038 & 0.000000000 \\
\hline $\mathrm{H}_{15}$ & -5.369668219 & -3.467074440 & -0.120384593 & $\mathrm{H}_{15}$ & -5.429315862 & -3.640996422 & 0.000000000 \\
\hline
\end{tabular}


Table SI3: Cartesian coordinates of conical intersections (in au; continued)

\begin{tabular}{|c|c|c|c|c|c|c|c|}
\hline & \multicolumn{3}{|c|}{$\left({ }^{1} \mathrm{~L}_{b} /{ }^{1} \mathrm{n} \pi^{*}\right)_{\mathrm{CI}}\left(C_{1}\right)$} & & \multicolumn{3}{|c|}{$\left({ }^{1} \mathrm{~L}_{b} /{ }^{1} \mathrm{n} \pi^{*}\right)_{\mathrm{CI}}\left(C_{s}\right)$} \\
\hline & $\mathrm{x}$ & $\mathrm{y}$ & $\mathrm{Z}$ & & $\mathrm{x}$ & $\mathrm{y}$ & $\mathrm{z}$ \\
\hline & \multicolumn{3}{|c|}{ gas } & & \multicolumn{3}{|c|}{ gas } \\
\hline $\mathrm{N}_{1}$ & -3.692017712 & 0.932141186 & -0.113650618 & $\mathrm{~N}_{1}$ & -3.745165794 & 0.822821239 & 0.000000000 \\
\hline $\mathrm{C}_{2}$ & -2.521507320 & 3.240081405 & 0.082219077 & $\mathrm{C}_{2}$ & -2.683499277 & 3.168998812 & 0.000000000 \\
\hline $\mathrm{N}_{3}$ & 0.026564533 & 3.631494076 & 0.024868889 & $\mathrm{~N}_{3}$ & -0.117708156 & 3.589817216 & 0.000000000 \\
\hline $\mathrm{C}_{4}$ & 1.346392454 & 1.491558657 & -0.019566601 & $\mathrm{C}_{4}$ & 1.318652934 & 1.517343180 & 0.000000000 \\
\hline $\mathrm{C}_{5}$ & 0.364807108 & -1.047689146 & -0.137294228 & $\mathrm{C}_{5}$ & 0.396664762 & -1.026292603 & 0.000000000 \\
\hline $\mathrm{C}_{6}$ & -2.315964364 & -1.292681179 & -0.245935096 & $\mathrm{C}_{6}$ & -2.250255974 & -1.311379718 & 0.000000000 \\
\hline $\mathrm{N}_{7}$ & 2.288045372 & -2.759594493 & -0.112042514 & $\mathrm{~N}_{7}$ & 2.371334739 & -2.698575622 & 0.000000000 \\
\hline $\mathrm{C}_{8}$ & 4.371543289 & -1.427573184 & 0.036916991 & $\mathrm{C}_{8}$ & 4.422722121 & -1.313031526 & 0.000000000 \\
\hline $\mathrm{N}_{9}$ & 3.921204000 & 1.151066118 & 0.105207479 & $\mathrm{~N}_{9}$ & 3.912373188 & 1.259847281 & 0.000000000 \\
\hline $\mathrm{N}_{10}$ & -3.599955995 & -3.530029550 & 0.336680971 & $\mathrm{~N}_{10}$ & -3.442948040 & -3.607699443 & 0.000000000 \\
\hline $\mathrm{H}_{11}$ & -3.740076093 & 4.866506084 & 0.168906310 & $\mathrm{H}_{11}$ & -3.951310197 & 4.779438193 & 0.000000000 \\
\hline $\mathrm{H}_{12}$ & 6.238188213 & -2.192154813 & 0.092012055 & $\mathrm{H}_{12}$ & 6.313942599 & -2.028587651 & 0.000000000 \\
\hline $\mathrm{H}_{13}$ & 5.206521836 & 2.508687648 & 0.233235363 & $\mathrm{H}_{13}$ & 5.156757179 & 2.654125517 & 0.000000000 \\
\hline $\mathrm{H}_{14}$ & -2.563684353 & -5.063271750 & -0.040966621 & $\mathrm{H}_{14}$ & -2.403277831 & -5.172514820 & 0.000000000 \\
\hline \multirow[t]{2}{*}{$\mathrm{H}_{15}$} & -5.291866231 & -3.645241255 & -0.498698542 & $\mathrm{H}_{15}$ & -5.313801653 & -3.730387094 & 0.000000000 \\
\hline & \multicolumn{3}{|c|}{$\mathrm{CH}_{3} \mathrm{CN}$} & & \multicolumn{3}{|c|}{$\mathrm{CH}_{3} \mathrm{CN}$} \\
\hline $\mathrm{N}_{1}$ & -3.676883642 & 0.952924200 & -0.026828760 & $\mathrm{~N}_{1}$ & -3.747710272 & 0.828212615 & 0.000000000 \\
\hline $\mathrm{C}_{2}$ & -2.519528658 & 3.253861306 & 0.081126721 & $\mathrm{C}_{2}$ & -2.681055398 & 3.172335020 & 0.000000000 \\
\hline $\mathrm{N}_{3}$ & 0.031114470 & 3.629404877 & -0.030886128 & $\mathrm{~N}_{3}$ & -0.109251422 & 3.588169887 & 0.000000000 \\
\hline $\mathrm{C}_{4}$ & 1.361535062 & 1.478053262 & -0.038021426 & $\mathrm{C}_{4}$ & 1.329272518 & 1.513943530 & 0.000000000 \\
\hline $\mathrm{C}_{5}$ & 0.365665518 & -1.043496807 & -0.081430401 & $\mathrm{C}_{5}$ & 0.395015468 & -1.021130836 & 0.000000000 \\
\hline $\mathrm{C}_{6}$ & -2.320321875 & -1.272764424 & -0.161216644 & $\mathrm{C}_{6}$ & -2.258626840 & -1.306941562 & 0.000000000 \\
\hline $\mathrm{N}_{7}$ & 2.277766426 & -2.770746619 & -0.048522204 & $\mathrm{~N}_{7}$ & 2.366788585 & -2.703484111 & 0.000000000 \\
\hline $\mathrm{C}_{8}$ & 4.374536589 & -1.432638287 & 0.037390585 & $\mathrm{C}_{8}$ & 4.420162320 & -1.316272889 & 0.000000000 \\
\hline $\mathrm{N}_{9}$ & 3.935706906 & 1.131720928 & 0.069226489 & $\mathrm{~N}_{9}$ & 3.916759267 & 1.249949996 & 0.000000000 \\
\hline $\mathrm{N}_{10}$ & -3.641355014 & -3.540917462 & 0.226105293 & $\mathrm{~N}_{10}$ & -3.449559595 & -3.600866844 & 0.000000000 \\
\hline $\mathrm{H}_{11}$ & -3.727321015 & 4.888159326 & 0.159654444 & $\mathrm{H}_{11}$ & -3.940349365 & 4.786413731 & 0.000000000 \\
\hline $\mathrm{H}_{12}$ & 6.240070982 & -2.199336230 & 0.080328317 & $\mathrm{H}_{12}$ & 6.312813729 & -2.027713804 & 0.000000000 \\
\hline $\mathrm{H}_{13}$ & 5.249883448 & 2.478037755 & 0.085254113 & $\mathrm{H}_{13}$ & 5.183923585 & 2.632819774 & 0.000000000 \\
\hline $\mathrm{H}_{14}$ & -2.602883025 & -5.032897926 & -0.298624078 & $\mathrm{H}_{14}$ & -2.419984808 & -5.172932294 & 0.000000000 \\
\hline $\mathrm{H}_{15}$ & -5.269680662 & -3.577369534 & -0.742573892 & $\mathrm{H}_{15}$ & -5.321413405 & -3.732308478 & 0.000000000 \\
\hline \multicolumn{4}{|c|}{$\mathrm{H}_{2} \mathrm{O}$} & & \multicolumn{3}{|c|}{$\mathrm{H}_{2} \mathrm{O}$} \\
\hline $\mathrm{N}_{1}$ & -3.695555399 & 0.952525440 & 0.033791668 & $\mathrm{~N}_{1}$ & -3.740447601 & 0.835637648 & 0.000000000 \\
\hline $\mathrm{C}_{2}$ & -2.475582499 & 3.256092112 & -0.019502283 & $\mathrm{C}_{2}$ & -2.682541795 & 3.184833012 & 0.000000000 \\
\hline $\mathrm{N}_{3}$ & 0.062648132 & 3.644244300 & -0.064283066 & $\mathrm{~N}_{3}$ & -0.097069895 & 3.573485519 & 0.000000000 \\
\hline $\mathrm{C}_{4}$ & 1.367627143 & 1.489737691 & -0.013659970 & $\mathrm{C}_{4}$ & 1.337276299 & 1.496837572 & 0.000000000 \\
\hline $\mathrm{C}_{5}$ & 0.322462957 & -1.032856710 & 0.143074782 & $\mathrm{C}_{5}$ & 0.392541312 & -1.022132692 & 0.000000000 \\
\hline $\mathrm{C}_{6}$ & -2.323424097 & -1.255179949 & 0.301892773 & $\mathrm{C}_{6}$ & -2.273853457 & -1.303473254 & 0.000000000 \\
\hline $\mathrm{N}_{7}$ & 2.254752942 & -2.777745496 & 0.120985140 & $\mathrm{~N}_{7}$ & 2.376935163 & -2.707104099 & 0.000000000 \\
\hline $\mathrm{C}_{8}$ & 4.339647115 & -1.448989988 & -0.052568168 & $\mathrm{C}_{8}$ & 4.431571761 & -1.311165723 & 0.000000000 \\
\hline $\mathrm{N}_{9}$ & 3.962052668 & 1.112877545 & -0.036869641 & $\mathrm{~N}_{9}$ & 3.922111586 & 1.237063465 & 0.000000000 \\
\hline $\mathrm{N}_{10}$ & -3.610837565 & -3.583030878 & -0.128179693 & $\mathrm{~N}_{10}$ & -3.479237155 & -3.581526190 & 0.000000000 \\
\hline $\mathrm{H}_{11}$ & -3.703970255 & 4.888251054 & -0.114196335 & $\mathrm{H}_{11}$ & -3.925185662 & 4.803994324 & 0.000000000 \\
\hline $\mathrm{H}_{12}$ & 6.201423638 & -2.242100752 & -0.192922073 & $\mathrm{H}_{12}$ & 6.326235556 & -2.022728975 & 0.000000000 \\
\hline $\mathrm{H}_{13}$ & 5.199016605 & 2.347659845 & -0.746205714 & $\mathrm{H}_{13}$ & 5.202236263 & 2.613919153 & 0.000000000 \\
\hline $\mathrm{H}_{14}$ & -2.711217269 & -4.690606981 & -1.404972441 & $\mathrm{H}_{14}$ & -2.495507085 & -5.183736260 & 0.000000000 \\
\hline $\mathrm{H}_{15}$ & -5.351854171 & -3.308446596 & -0.881556496 & $\mathrm{H}_{15}$ & -5.353870355 & -3.702694997 & 0.000000000 \\
\hline
\end{tabular}



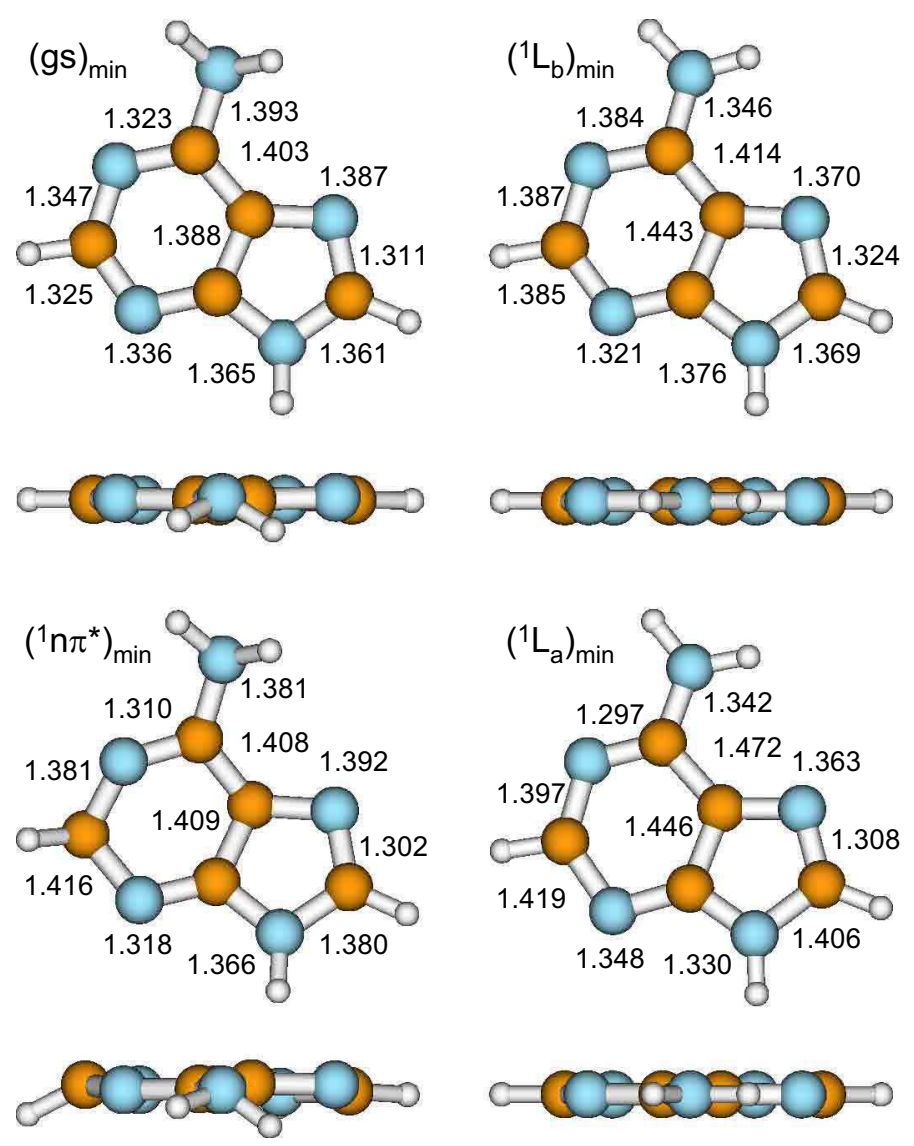

Figure SI1: Optimized geometries of ground and excited-state energy minima in aqueous solution. Bond lengths are in angstroms. 